\author{
В.Г. Падалко \\ Дніпровський національний університет імені Олеся Гончара
}

\title{
СТРУКТУРА ТА ОСНОВНІ НАПРЯМИ РОЗВИТКУ МАТЕМАТИЧНОЇ ТЕОРІЇ ОПТИМАЛЬНОГО РОЗБИТТЯ МНОЖИН
}

Викладено структуру та основні напрями математичної теорії оптимального розбиття множин, наведено приклади застосування результатів цієї теорії до розв'язання широкого спектру різних за свосю природою теоретичних і практичних оптимізаційних задач, які зводяться до неперервних моделей оптимального розбиття множин. Показано можливість та переваги застосування теорії оптимального розбиття множин до побудови діаграми Вороного та її узагальнень.

Ключові слова: нескінченновимірне математичне програмування, теорія оптимального розбиття множин, розпізнавання образів, недиференційовна оптимізація, нечітка діаграма Вороного.

\author{
V.G. Padalko \\ Oles Honchar Dnipro National University
}

\section{STRUCTURE AND MAIN DIRECTIONS OF DEVELOPMENT OF THE MATHEMATICAL THEORY OF OPTIMAL SET PARTITIONING}

The paper provides the structure of the mathematical theory of continuous problems of optimal partitioning of sets from $\boldsymbol{n}$-dimensional Euclidean space, which are nonclassical problems of infinite-dimensional mathematical programming with Boolean variables. Currently, a number of directions have been developed in the theory of optimal set partitioning, which are due to various types of mathematical formulations of partitioning problems. They can be linear and nonlinear; single-product and multi-product; deterministic and stochastic; under conditions of complete and incomplete information about the initial data; static and dynamic; with constraints and without them; both with a given position of the centers of subsets, and with finding the optimal variant of their location.

The mathematical theory of optimal set partitioning is based on a unified approach consisting in reducing the original infinite-dimensional optimization problems in a certain way (for example, using the Lagrange functional) to non-smooth, as a rule, finite-dimensional optimization problems, for the numerical solution of which modern efficient methods of non-differentiable optimization - variations of Shor's algorithm - are applied. A feature of this approach is the fact that the solution of the original infinite-dimensional optimization problems can be obtained analytically in an explicit form, and the analytical expression includes the parameters sought as the optimal solution of the abovementioned auxiliary finite-dimensional optimization problems with non-smooth objective functions.

The theory of optimal set partitioning is an actively developing area of modern optimization theory and is effectively used to solve a wide range of theoretical and practical classes of optimization problems that are different in nature, which are reduced in a mathematical setting to continuous models of optimal set partitioning. These are the pattern recognition problems (clear and fuzzy) to minimize the average loss function from

(C) Падалко В.Г., 2021 
incorrect recognition; problems of the theory of classification and clustering, which investigates the problems of partitioning of a given set (finite-dimensional or infinitedimensional) of elements into disjoint subsets. These are also problems arising in the theory of statistical decisions when partitioning the feature space into disjoint classes (generalized Neumann-Pearson problem) and many others.

The paper shows the possibility and advantages of applying the mathematical theory of optimal set partitioning to the construction of a Voronoi diagram and its various generalizations. The result of using the theory of optimal set partitioning as a universal mathematical apparatus for constructing a Voronoi diagram and its generalizations demonstrates the ability to construct not only already known Voronoi diagrams, but also new ones.

Keywords: infinite-dimensional mathematical programming, theory of optimal set partitioning, pattern recognition, undifferentiated optimization, fuzzy Voronoi diagram.

\section{В.Г. Падалко \\ Днипровский национальный университет имени Олеся Гончара \\ СТРУКТУРА И ОСНОВНЫЕ НАПРАВЛЕНИЯ РАЗВИТИЯ МАТЕМАТИЧЕСКОЙ ТЕОРИИ ОПТИМАЛЬНОГО РАЗБИЕНИЯ МНОЖЕСТВ}

Изложены структура и основные направления математической теории оптимального разбиения множеств, приведены примеры применения результатов этой теории к решению широкого спектра различных по своей природе теоретических и практических оптимизационных задач, которые сводятся к непрерывным моделям оптимального разбиения множеств. Показана возможность и преимущества применения теории оптимального разбиения множеств к построению диаграммы Вороного и ее обобщений.

Ключевые слова: бесконечномерное математическое программирование, теория оптимального разбиения множеств, распознавание образов, недифференцированная оптимизация, нечеткая диаграмма Вороного.

Вступ. Основи математичної теорії оптимального розбиття множин (ОРМ), що є новим (некласичним) розділом нескінченновимірного математичного програмування були закладені членом-кореспондентом Національної академії наук України, доктором фізико-математичних наук, професором O.M. Кісельовою більш ніж 60 років тому $[1,2,3]$. На теперішній час в теорії неперервних задач ОРМ сформувався ряд напрямів, обумовлених як різними типами математичних постановок задач розбиття, так і різними сферами її застосувань.

Метою дослідження є аналіз сучасної структури математичної теорії оптимального розбиття множин та основних напрямів їі розвитку.

1 Матеріали і методи. Розробка математичної теорії оптимального розбиття множин починалась 3 розв'язання спрощеної моделі нескінченновимірної задачі розміщення виробництва, що називається в зарубіжній науковій літературі задачею location-allocation, в такій постановці [3].

Нехай споживач деякої однорідної продукції рівномірно розподілений в області $\Omega \subset E_{2}$. Скінченне число $N$ виробників цієї продукції розташовані в 
ізольованих точках $\tau_{i}=\left(\tau_{i}^{(1)}, \tau_{i}^{(2)}\right), \quad i=1, \ldots, N$, області $\Omega$. Вважаються заданими: $\rho(x)$ - попит на продукцію споживача 3 координатами $x=\left(x^{(1)}, x^{(2)}\right) ; c\left(x, \tau_{i}\right)-$ вартість транспортування одиниці продукції від виробника $\tau_{i}=\left(\tau_{i}^{(1)}, \tau_{i}^{(2)}\right)$ споживачеві 3 координатами $x=\left(x^{(1)}, x^{(2)}\right)$. Передбачається, що прибуток виробника залежить тільки від його витрат, які $\epsilon$ сумою виробничих і транспортних витрат.

Для кожного $i$-го виробника задана функція $\varphi_{i}\left(Y_{i}\right)$, що описує залежність вартості виробництва від його потужності $Y_{i}$, яка визначається за формулою $Y_{i}=\int_{\Omega_{i}} \rho(x) d x$, і наведені капітальні витрати на реконструкцію $i$-го виробника для збільшення його потужності від існуючої до проектної $Y_{i}$.

Множину споживачів $\Omega$ можна розбивати на зони обслуговування $\Omega_{i}$ споживачів $i$-м виробником так, щоб

$$
\bigcup_{i=1}^{N} \Omega_{i}=\Omega, \text { mes }\left(\Omega_{i} \cap \Omega_{j}\right)=0, i \neq j, i, j=1, \ldots, N .
$$

При цьому потужність $i$-го виробника визначається сумарним попитом споживачів, що належать $\Omega_{i}$, і не перевищує заданих обсягів:

$$
\int_{\Omega_{i}} \rho(x) d x \leq b_{i}, i=1, \ldots, N,
$$

не виключається, що деякі з підмножин $\Omega_{i}$ виявляться порожніми.

Потрібно розбити множину споживачів $\Omega$ на зони обслуговування їх $N$ виробниками, тобто на підмножини $\Omega_{i}, i=1, \ldots, N$, і розмістити цих виробників в $\Omega$ так, щоб мінімізувати функціонал сумарних витрат на виробництво продукції і доставку ії до споживачів:

$$
F\left(\left\{\Omega_{1}, \ldots, \Omega_{N}\right\},\left\{\tau_{1}, . ., \tau_{N}\right\}\right)=\sum_{i=1}^{N}\left\{\int_{\Omega_{i}} c\left(x, \tau_{i}\right) \rho(x) d x+\varphi_{i}\left(\int_{\Omega_{i}} \rho(x) d x\right)\right\}
$$

за умов (1), (2).

Задача (1)-(3) $є$ нескінченновимірною задачею розміщення.

У більшості практичних задач вартість виробництва продукції на промисловому підприємстві потужності $Y_{i}$ дорівнює добутку собівартості цієї продукції на ії кількість. В силу цього маємо:

$$
\varphi_{i}\left(Y_{i}\right)=d_{i}+a_{i} Y_{i}, i=1, \ldots, N \text {. }
$$

Підставляючи вираз (4) в (3) отримаємо

$$
F\left(\left\{\Omega_{1}, \ldots, \Omega_{N}\right\},\left\{\tau_{1}, . ., \tau_{N}\right\}\right)=\sum_{i=1}^{N} \int_{\Omega_{i}}\left(c\left(x, \tau_{i}\right)+a_{i}\right) \rho(x) d x .
$$


Задача (1)-(5) при $a_{i}=0, i=1, \ldots, N, \epsilon$ нескінченновимірною транспортною задачею.

Олена Михайлівна Кісельова отримала необхідні умови оптимальності для задачі (1), (5) у вигляді такої теореми і наслідку з неї [2].

Теорема. Якщо можливе розбиття $\left(\Omega_{* 1}, \ldots, \Omega_{*_{i}}, \ldots, \Omega_{*_{N}}\right) \in$ оптимальним для задачі (1), (5), то

$c\left(x, \tau_{i}\right)+a_{i} \leq c\left(x, \tau_{k}\right)+a_{k}$ майже всюди (м.в.) для $x \in \Omega_{*_{i}}, i, k=1, \ldots, N$. (6)

Наслідок. В точках $x$, які належать оптимальній границі між підмножинами $\Omega_{*_{i}}$ і $\Omega_{*_{k}}$, в нерівності (6) досягається знак рівності.

\section{2 Результати.}

\section{1 Загальна структура теорії оптимального розбиття множин}

В математичній теорії неперервних задач ОРМ до теперішнього часу сформувався ряд напрямів, обумовлених як різними типами математичних постановок задач розбиття, так і різними сферами їі застосувань. Наведемо деякі 3 цих напрямів і посилання на основні для цієї теорії роботи. Це:

- детерміновані лінійні і нелінійні, однопродуктові і багатопродуктові задачі ОРМ при обмеженнях, як із заданим положенням центрів підмножин, так і з відшуканням оптимального варіанту їх розташування, [4-10];

- задачі оптимального розбиття множин в умовах невизначеності, для зняття невизначеності в яких пропонується застосовувати або математичний апарат стохастичного нескінченновимірного математичного програмування (якщо частина інформації має ймовірнісний характер), або апарати нечітких множин і нечіткої логіки (якщо параметри, що входять до опису моделей, $є$ нечіткими, неточними, недостовірними тощо), [11-16];

- динамічні задачі оптимального розбиття з критерієм оптимальності, що залежить від фазових траєкторій і управління деякої заданої керованої системи, $[17,18]$;

- неперервні задачі про кульове покриття, що зводяться до задач ОРМ, [19, 20].

- задачі оптимального розбиття множин (як в умовах визначеності, так i невизначеності) в ускладнених постановках, а саме: 3 додатковими обмеженнями на розташування центрів підмножин, двоетапні неперервно-дискретної задачі розміщення-розбиття [21-25].

Структуру сформованої до теперішнього часу теорії оптимального розбиття множин можна представити у вигляді блок-схеми 1. 


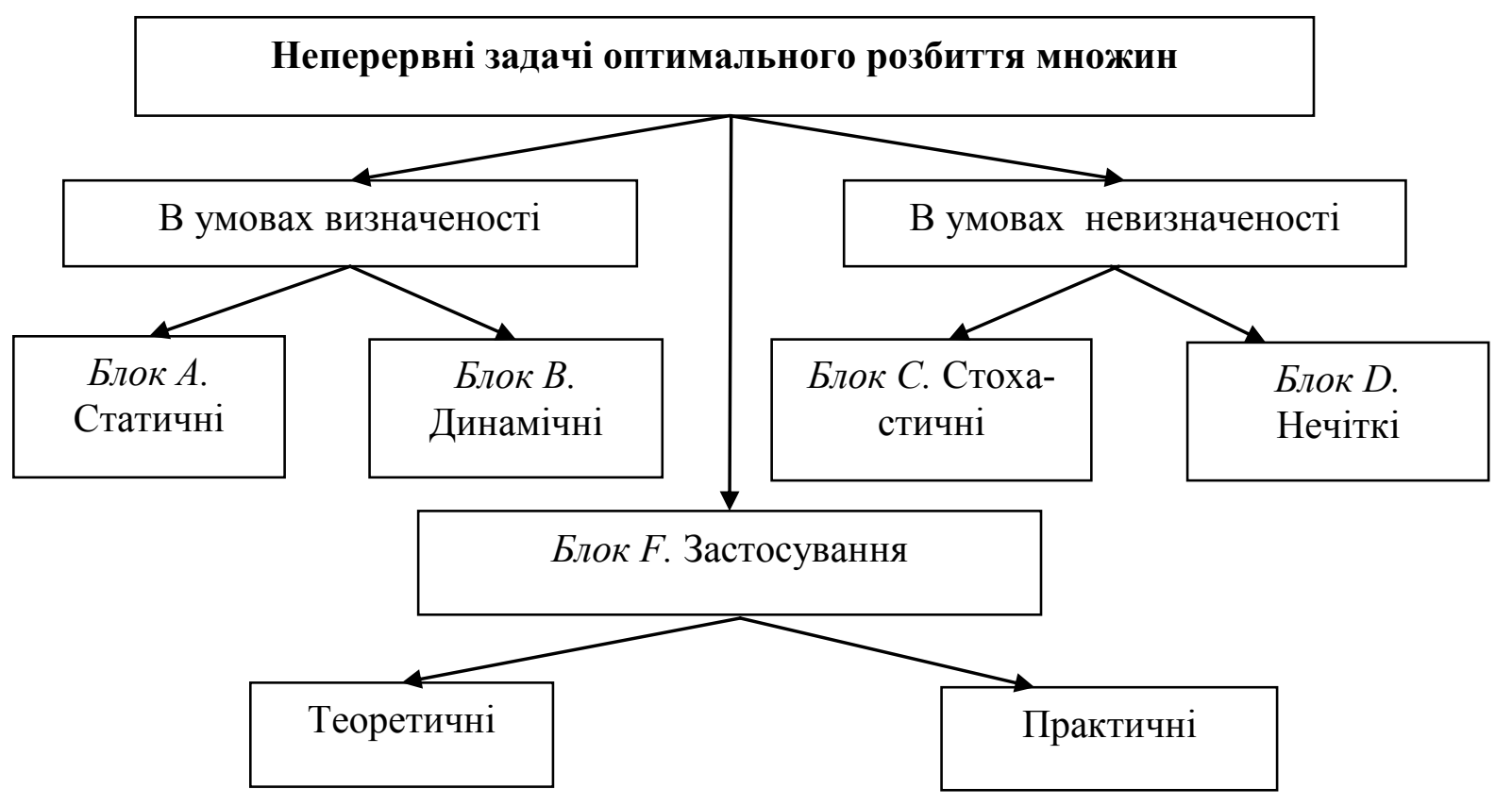

Рис. 1. Структура теорії оптимального розбиття множин

\section{2 Класифікація статичних і динамічних задач ОРМ}

Найбільш вивченими на сьогоднішній день $є$ статичні задачі оптимального розбиття множин (Блок А). У блоці В розглядаються динамічні задачі ОРМ 3 критерієм оптимальності, що залежать від фазових траєкторій та управлінь деякої заданої керованої системи. Так, наприклад, у задачі (1), (2), (5), яка розглядалась як нескінченновимірний аналог транспортної задачі, функції попиту і вартості транспортування одиниці продукції можуть змінюватися протягом деякого проміжку часу в силу дії певних економічних факторів і $є$ невідомими заздалегідь фазовими траєкторіями заданого керованого процесу, який описується деякою системою диференціальних рівнянь 3 початковими умовами.

Для розв'язання наведених у блоках А і В класів задач ОРМ запропонований, як уже зазначалося вище, єдиний підхід, в основі якого лежить наступна ідея. Вихідні задачі ОРМ, які математично сформульовані як нескінченновимірні задачі оптимізації, зводяться певним чином (наприклад, через функціонал Лагранжа) до допоміжних скінченновимірних негладких задач максимізації або негладких задач максиміна, для чисельного розв'язання яких застосовуються сучасні ефективні методи недиференційовної оптимізації, а саме, різні модифікації $r$-алгоритму Н.3. Шора [26, 27].

Особливістю такого підходу для лінійних задач ОРМ $є$ той факт, що розв'язання вихідних нескінченновимірних задач оптимізації вдається отримати аналітично в явному вигляді, причому в аналітичний вираз можуть входити параметри, що відшукуються як оптимальний розв'язок вищеназваних допоміжних скінченновимірних задач оптимізації з негладкими цільовими функціями. Розв'язок нелінійних задач ОРМ вдається отримати у вигляді операторних рівнянь 3 параметрами, що відшукуються як оптимальний 
розв'язок допоміжних скінченновимірних негладких задач оптимізації, для розв'язання яких застосовуються різні модифікації $r$-алгоритму.

Більш детально блоки А і В представимо у вигляді такої блок-схеми:

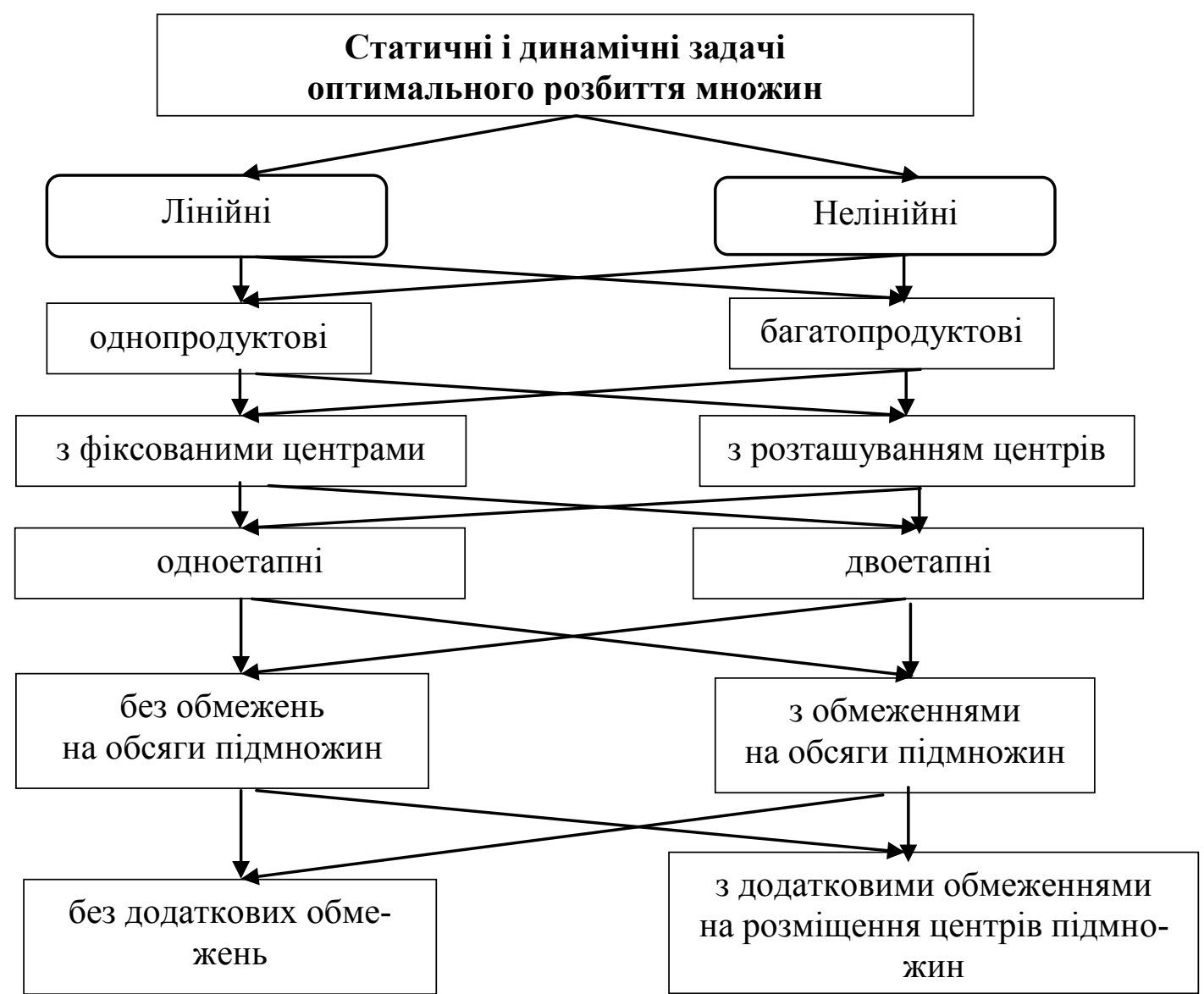

Рис. 2. Класифікація неперервних задач ОРМ (блоки А і В)

Далі наведемо математичну постановку однієї задачі оптимального розбиття з блоку А, що є узагальненням задачі (1), (2), (5), а саме постановку лінійної однопродуктовій задачі А1 оптимального розбиття множин при обмеженнях у формі рівностей та нерівностей з відшуканням координат центрів підмножин.

Нехай $\Omega$ - обмежена, вимірна за Лебегом множина $3 n$-вимірного евклідового простору $E_{n}$. Сукупність вимірних за Лебегом підмножин $\Omega_{1}, \ldots, \Omega_{N}$ множини $\Omega \subset \mathrm{E}_{n}$ назвемо можливим розбиттям множини $\Omega$ на ії підмножини, що не перетинаються, $\Omega_{1}, \ldots, \Omega_{N}$, якщо $\bigcup_{i=1}^{N} \Omega_{i}=\Omega, \operatorname{mes}\left(\Omega_{i} \cap \Omega_{j}\right)=0$, $i \neq j, i, j=1, \ldots, N$, де $\operatorname{mes}(\cdot)$ означає міру Лебега. Позначимо клас усіх можливих розбиттів множини $\Omega$ на підмножини, що не перетинаються $\Omega_{1}, \ldots, \Omega_{N}$, через $\sum_{\Omega}^{N}$, тобто

$$
\sum_{\Omega}^{N}=\left\{\left(\Omega_{1}, \ldots, \Omega_{N}\right): \bigcup_{i=1}^{N} \Omega_{i}=\Omega, \operatorname{mes}\left(\Omega_{i} \cap \Omega_{j}\right)=0, i \neq j, i, j=1, \ldots, N\right\} .
$$

Введемо функціонал 
ISSN 2074-5893 Питання прикладної математики і математичного моделювання. Випуск 21

$$
F\left(\left\{\Omega_{1}, \ldots, \Omega_{N}\right\},\left\{\tau_{1}, \ldots, \tau_{N}\right\}\right)=\sum_{i=1}^{N} \int_{\Omega_{i}}\left(c\left(x, \tau_{i}\right)+a_{i}\right) \rho(x) d x
$$

Під неперервною лінійною однопродуктовою задачею оптимального розбиття множини $\Omega$ з п-вимірного евклідового простору $E_{n}$ на підмножини $з$ відшуканням координат иентрів (з розташуванням иентрів) підмножин при обмеженнях у формі рівностей та нерівностей будемо розуміти задачу А1.

Задача Al [10]. Знайти $\min _{\left\{\Omega_{1}, \ldots, \Omega_{N}\right\},\left\{\tau_{1}, \ldots, \tau_{N}\right\}} F\left(\left\{\Omega_{1}, \ldots, \Omega_{N}\right\},\left\{\tau_{1}, \ldots, \tau_{N}\right\}\right)$

за умов

$$
\begin{gathered}
\int_{\Omega_{i}} \rho(x) d x=b_{i}, \quad i=1, \ldots, p, \quad \int_{\Omega_{i}} \rho(x) d x \leq b_{i}, \quad i=p+1, . ., N, \\
\left\{\Omega_{1}, \ldots, \Omega_{N}\right\} \in \Sigma_{\Omega}^{N}, \quad \tau=\left(\tau_{1}, \ldots, \tau_{N}\right) \in \Omega^{N},
\end{gathered}
$$

де $x=\left(x^{(1)}, \ldots, x^{(n)}\right) \in \Omega ; \tau_{i}=\left(\tau_{i}^{(1)}, \ldots, \tau_{i}^{(n)}\right) \in \Omega$; функції $c\left(x, \tau_{i}\right)-$ обмежені, визначені на $\Omega \times \Omega$, вимірні за аргументом $x$ для будь-якого фіксованого $\tau_{i}=\left(\tau_{i}^{(1)}, \ldots, \tau_{i}^{(n)}\right)$ з $\Omega$ для усіх $i=1, . ., N$; координати $\tau_{i}^{(1)}, \ldots, \tau_{i}^{(n)}$ центру $\tau_{i}$, $i=1, . ., N$, заздалегідь невідомі; $\rho(x)$ - обмежена, вимірна, невід'ємна на $\Omega$ функція; $a_{1}, \ldots a_{N}, b_{1}, \ldots, b_{N}$ - задані невід'ємні числа, причому виконуються умови розв'язності задачі

$$
S=\int_{\Omega} \rho(x) d x \leq \sum_{\mathrm{i}=1}^{\mathrm{N}} b_{i}, 0 \leq b_{i} \leq S, i=1, \ldots, N .
$$

Тут і надалі інтеграли розуміються в сенсі Лебега. Будемо вважати, що міра множини граничних точок $\Omega_{i}, i=1, . ., N$, дорівнює нулю.

Розбиття $\left\{\Omega_{*_{1}}, \ldots, \Omega_{*_{N}}\right\}$, що є розв'язком задачі А1називають оптимальним.

Окремими випадками розглянутої задачі $\mathrm{A} 1 \epsilon$ задача ОРМ як із заданими координатами центрів підмножин, так і з невідомими заздалегідь; як без обмежень, так і з обмеженнями (див. рис. 2).

Наведемо приклади постановок таких задач, в яких всі умови на функції, що входять до цих постановок, залишаються такими ж, як і в задачі A1.

Задача A1.1. Неперервна задача оптимального розбиття множини $\Omega$ из $E_{n}$ на її підмножини $\Omega_{1}, \ldots, \Omega_{N}$, що не перетинаються, (серед яких можуть бути і порожні) без обмежень із заданими координатами центрів $\tau_{1}, \ldots, \tau_{N}$ підмножин $\Omega_{1}, \ldots, \Omega_{N}$, відповідно. Знайти $\min _{\left\{\Omega_{1}, \ldots, \Omega_{N}\right\} \in \Sigma_{\Omega}^{N}} F\left(\left\{\Omega_{1}, \ldots, \Omega_{N}\right\}\right)$, де $F\left(\left\{\Omega_{1}, \ldots, \Omega_{N}\right\}\right)=\sum_{i=1}^{N} \int_{\Omega_{i}}\left(c\left(x, \tau_{i}\right)+a_{i}\right) \rho(x) d x$; $x=\left(x^{(1)}, \ldots, x^{(n)}\right) \in \Omega ; \tau=\left(\tau_{1}, \ldots, \tau_{i}, \ldots, \tau_{N}\right) \in \Omega^{N} ; a_{1}, \ldots a_{N}$ - задані числа, координати $\tau_{i}^{(1)}, \ldots, \tau_{i}^{(n)}$ центру $\tau_{i}, i=1, \ldots, N$, фіксовані.

Задача A1.2. Неперервна задача оптимального розбиття множини $\Omega 3$ $E_{n}$ на їі підмножини $\Omega_{1}, \ldots, \Omega_{N}$ що не перетинаються, (серед яких можуть бути i 
порожні) без обмежень із розміщенням цеентрів $\tau_{1}, \ldots, \tau_{N}$ підмножин $\Omega_{1}, \ldots, \Omega_{N}$ відповідно. Знайти

$$
\min _{\left\{\Omega_{1}, \ldots, \Omega_{N}\right\},\left\{\tau_{1}, \ldots, \tau_{N}\right\}} F\left(\left\{\Omega_{1}, \ldots, \Omega_{N}\right\},\left\{\tau_{1}, \ldots, \tau_{N}\right\}\right),\left\{\Omega_{1}, \ldots, \Omega_{N}\right\} \in \Sigma_{\Omega}^{N}, \tau=\left(\tau_{1}, \ldots, \tau_{N}\right) \in \Omega^{N},
$$

де $F\left(\left\{\Omega_{1}, \ldots, \Omega_{N}\right\},\left\{\tau_{1}, \ldots, \tau_{N}\right\}\right)=\sum_{i=1}^{N} \int_{\Omega_{i}}\left(c\left(x, \tau_{i}\right)+a_{i}\right) \rho(x) d x, \quad x=\left(x^{(1)}, \ldots, x^{(n)}\right) \in \Omega$; $\tau_{i}=\left(\tau_{i}^{(1)}, \ldots, \tau_{i}^{(n)}\right) \in \Omega$; координати $\tau_{i}^{(1)}, \ldots, \tau_{i}^{(n)}$ центру $\tau_{i}, i=1, . ., N$, заздалегідь невідомі (необхідно знайти).

Задача A1.3. Неперервна задача оптимального розбиття множини $\Omega 3$ $E_{n}$ на ії підмножини $\Omega_{1}, \ldots, \Omega_{N}$ що не перетинаються, (серед яких можуть бути і порожні) при обмеженнях у формі рівностей та нерівностей із заданими координатами центрів $\tau_{1}, \ldots, \tau_{N}$ підмножин $\Omega_{1}, \ldots, \Omega_{N}$, відповідно. Знайти

$$
\min _{\left\{\Omega_{1}, \ldots, \Omega_{N}\right\}} F\left(\left\{\Omega_{1}, \ldots, \Omega_{N}\right\}\right),\left\{\Omega_{1}, \ldots, \Omega_{N}\right\} \in \Sigma_{\Omega}^{N}
$$

за умов

$$
\int_{\Omega_{i}} \rho(x) d x=b_{i}, \quad i=1, \ldots, p, \quad \int_{\Omega_{i}} \rho(x) d x \leq b_{i}, \quad i=p+1, . ., N,
$$

де

$$
F\left(\left\{\Omega_{1}, \ldots, \Omega_{N}\right\}\right)=\sum_{i=1}^{N} \int_{\Omega_{i}}\left(c\left(x, \tau_{i}\right)+a_{i}\right) \rho(x) d x, \quad x=\left(x^{(1)}, \ldots, x^{(n)}\right) \in \Omega ;
$$

$\tau_{i}=\left(\tau_{i}^{(1)}, \ldots, \tau_{i}^{(n)}\right) \in \Omega ;$ координати $\tau_{i}^{(1)}, \ldots, \tau_{i}^{(n)}$ центру $\tau_{i}, i=1, . ., N$, фіксовані; $a_{1}, \ldots a_{N}, b_{1}, \ldots, b_{N}$ - задані невід'ємні числа, причому виконуються умови розв'язності задачі

$$
S=\int_{\Omega} \rho(x) d x \leq \sum_{i=1}^{N} b_{i}, \quad 0 \leq b_{i} \leq S, \quad i=1, \ldots, N .
$$

\section{3 Класифікація нечітких задач ОРМ}

Відзначимо, що переважна більшість задач теорії ОРМ досліджувалась в умовах визначеності. Однак реальні ситуації, для яких створюються моделі оптимального розбиття множин, найчастіше характеризуються деяким ступенем невизначеності: в початкових даних, в умовах і цілях. У цих випадках якість прийнятих рішень в оптимізаційних моделях розбиття множин знаходиться в прямій залежності від повноти урахування всіх невизначених факторів, істотних для наслідків від прийнятих рішень. Природним представляється узагальнення моделей розбиття в умовах визначеності на випадок моделей в умовах невизначеності. Це блоки C i D на рис. 1. Стохастичні задачі оптимального розбиття множин (блок C), у яких для врахування впливу невизначеності застосовується метод, що використовує лише деякі суб'єктивні імовірнісні характеристики параметрів задачі, досліджувалися у роботах $[18$, 20].

Клас задач оптимального розбиття множин, який пов'язаний 3 необхідністю врахування чинників невизначеності, що мають не ймовірнісностатистичну природу, відносять до нечітких задач оптимального розбиття 168 
множин. Це задачі ОРМ, в яких або окремі параметри, що входять до опису моделі, є нечіткими, неточними, недовизначеними, або є недостовірний математичний опис деяких залежностей в моделі (наприклад, функцій попиту та вартості транспортування одиниці продукції в нескінченновимірних транспортних задачах), або ж нечітко сформульовані самі критерії та (або) системи обмежень, або на модель оптимізації впливають зовнішні неконтрольовані збурення різного роду та ін. Для розкриття невизначеності в таких задачах (тобто для формалізації невизначеної інформації) застосовується апарат теоpiї нечітких множин, який базується на понятті нечіткої множини, введеного Л. А. Заде [28], а також апарат нечіткої логіки. Більш детальна класифікація нечітких задач ОРМ (блок D) у відповідності до того, у яких елементах задачі наявна нечіткість, представлена на рис. 3.

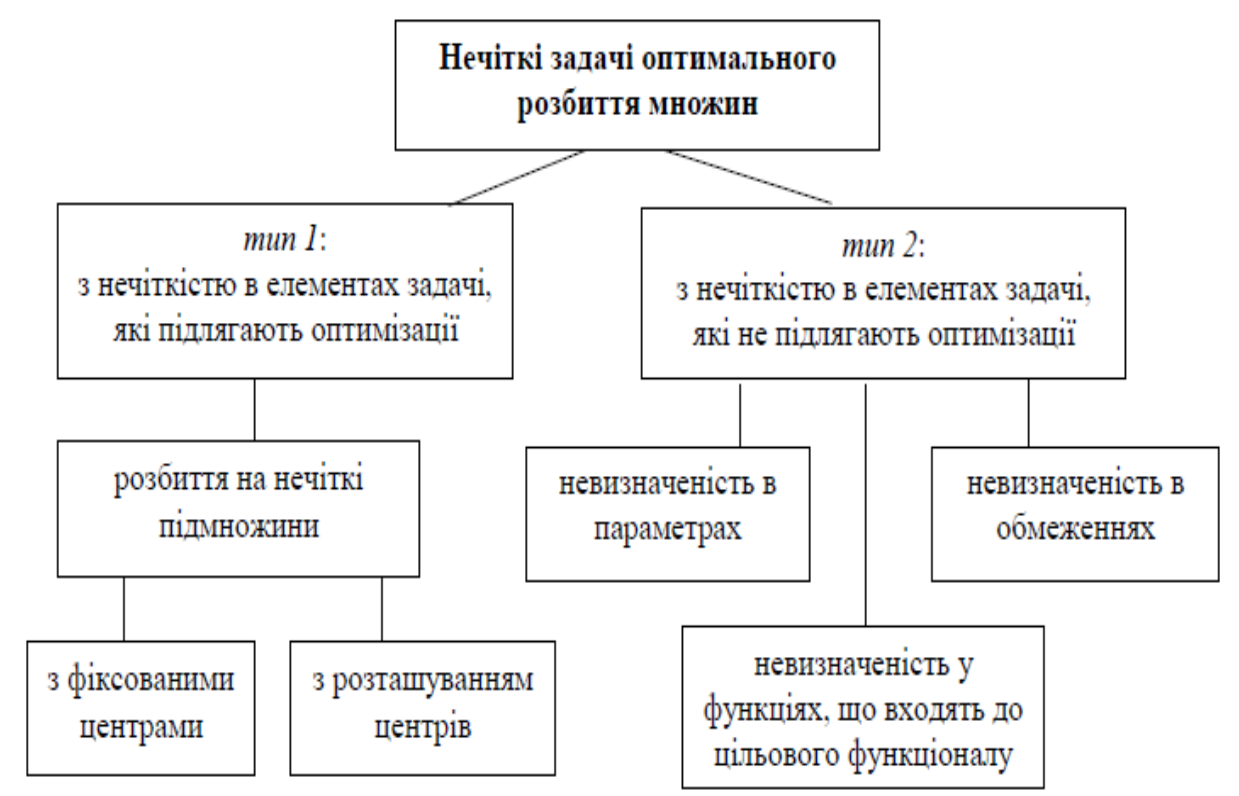

Рис. 3. Класифікація нечітких задач ОРМ

У класифікації нечітких задач ОРМ на рис. 3 виділено два основні типи нечітких задач ОРМ:

1) з нечіткістю в елементах задачі, які підлягають оптимізації;

2) з нечіткістю в елементах задачі, які не підлягають оптимізації.

Методи і алгоритми розв'язання задачі оптимального розбиття множини на нечіткі підмножини типу 1 із заданими координатами центрів підмножин розроблені у роботах [16], з відшуканням координат оптимального розміщення центрів цих підмножин - у роботах [21].

Для розв'язання задач типу 2 з нечіткістю в елементах задачі, які не підлягають оптимізації, розроблено єдиний підхід до розв'язання задач оптимального розбиття в умовах нечітких початкових даних, який базується на синтезі методів теорії оптимального розбиття множин та обчислювального інтелекту. Спочатку для зняття нечіткості у початкових даних застосовується метод 
нейролінгвістичної ідентифікації залежностей, а потім застосовується математичний та алгоритмічний апарати розв'язання задач ОРМ в умовах визначеності. Цим підтверджується універсальність теорії ОРМ, а саме, моделі і методи розв'язання неперервних задач оптимального розбиття можуть бути узагальнені на випадок, коли початкові дані задані нечітко. Єдиний підхід продемонстровано при розробці методів та алгоритмів розв'язання таких нечітких задач ОРМ: $з$ функцією у цільовому функціоналі, явний вид якої невідомий [12], з нечіткими параметрами в цільовому функціоналі [14], а також нескінченновимірної задачі розміщення-розбиття з нечіткими параметрами $[11,15]$.

Далі наведемо приклади математичних постановок нечітких задач ОРМ 3 блоку D.

Сформулюємо неперервну задачу оптимального розбиття множин $n$ вимірного евклидового простору $E_{n}$ на підмножини 3 нечітко описаними параметрами в цільовому функціоналі і обмеженнях (див. рис.3, тип 1).

Задача А2 [16]. (з нечітко описаними параметрами в иільовому функиіоналі і обмеження $)$. Знайти $\min _{\left\{\Omega_{1}, \ldots, \Omega_{\mathrm{N}}\right\} \in \sum_{\Omega}^{\mathrm{N}}} \sum_{i=1}^{N} \int_{\Omega_{i}}\left(c\left(x, \tau_{i}\right)+\theta_{i}\right) \rho\left(x, \theta_{0}\right) d x$

За умов $\int_{\Omega_{i}} \rho\left(x, \theta_{0}\right) d x \leq b_{i}, \quad i=1, \ldots, N$,

де $\Sigma_{\Omega}^{N}=\left\{\left(\Omega_{1}, \ldots, \Omega_{N}\right): \bigcup_{i=1}^{N} \Omega_{i}=\Omega, \operatorname{mes}\left(\Omega_{i} \cap \Omega_{j}\right)=0, i \neq j, i, j=1, \ldots, N\right\}$; $x=\left(x^{(1)}, \ldots, x^{(n)}\right) \in \Omega ; \tau_{i}=\left(\tau_{i}^{(1)}, \ldots, \tau_{i}^{(n)}\right) \in \Omega ; b_{1}, \ldots, b_{N}$ - задані позитивні числа. Тут $c\left(x, \tau_{i}\right)$ - функції, які обмежені, визначені на $\Omega \times \Omega \times R_{1}$, вимірні за $x$ на $\Omega$ при будь-яких фіксованих $\tau_{i} \in \Omega$ и $\theta_{i} \in R_{1} ; \rho\left(x, \theta_{0}\right)$ - невід'ємна, обмежена, вимірна за $x$ на $\Omega$ функція при будь-якому фіксованому $\theta_{0} 3 R_{1} ; \theta_{0}, \theta_{1}, \ldots, \theta_{N}$ - параметри, значення яких описані нечітко в формі нечітких підмножин універсальної множини $R_{1}$, які мають такий вигляд:

$$
\theta_{i}=\left\{\left(\theta_{i}, \mu\left(\theta_{i}\right)\right), \theta_{i} \in R_{1}, \mu_{i}: R_{1} \rightarrow[0,1]\right\}, \quad i=0,1, \ldots, N .
$$

Позначення «min» має сенс здійснення раціонального вибору розбиття $\left(\Omega_{1}, \ldots, \Omega_{N}\right)$, яке в певному сенсі «мінімізує» заданий цільовий функціонал.

Сформулюємо одну з можливих окремих постановок задачі 2 , в якій нечітко задані параметри в цільовому функціоналі.

Задача A2.1.

$$
\sum_{i=1}^{N} \int_{\Omega_{i}}\left(c\left(x, \tau_{i}\right) / \tilde{\omega}_{i}+\tilde{a}_{i}\right) \rho(x) d x \rightarrow \min _{\left(\left\{\Omega_{1}, \ldots, \Omega_{N}\right\}\right)}
$$

за умов

$$
\bigcup_{i=1}^{N} \Omega_{i}=\Omega, \operatorname{mes}\left(\Omega_{i} \cap \Omega_{j}\right)=0, i \neq j, i, j=1, \ldots, N,
$$

де $\tilde{a}_{i}, \tilde{\omega}_{i}, i=1, \ldots, N,-$ нечіткі параметри. 
Як вже зазначалося вище, вимога знаходження однозначного (чіткого) розбиття елементів множини $\Omega$ з $E_{n}$ може виявитися досить грубим і жорстким при розв'язанні задач з погано або слабо структурованою вихідною інформацією, тобто задач, в яких невизначеність має нечітко-можливісну природу. Ослаблення цієї вимоги здійснюється за рахунок введення в розгляд нечітких підмножин $\Omega_{i}, i=1, \ldots, N$ множини $\Omega$ і відповідних їм функцій належності, що приймають значення з відрізка $[0,1]$.

Тоді одним з варіантів нечіткої задачі ОРМ стає задача знаходження такого нечіткого розбиття множини $\Omega$ на його нечіткі підмножини, яке в певному сенсі «мінімізує» деякий цільової функціонал. Ця задача буде зведена до знаходження ступенів належності елементів множини $\Omega$ шуканим нечітким підмножинам $\Omega_{1}, \ldots, \Omega_{N}$, які в сукупності і визначають нечітке розбиття множини $\Omega$.

Для формулювання постановки задачі нечіткого оптимального розбиття наведемо відповідне означення з [10].

Означення. Нечітким розбиттям чіткої множини $\Omega$ з $E_{n}$, де $\Omega$ - обмежена, вимірна за Лебегом, опукла множина, назвемо систему нечітких підмножин $\mathfrak{R}(\Omega)=\left\{\Omega_{i}: \Omega_{i} \subseteq \Omega, \quad \forall i=\overline{1, n}\right\}$, де $\Omega=\left(\Omega, \mu_{\Omega}(x)\right), \mu_{\Omega}(x)=1, x \in \Omega$, для яких виконується умова: $\sum_{k=1}^{n} \mu_{k}(x)=\mu_{\Omega}(x)=1, \quad \forall x \in \Omega$.

Позначимо клас всіх можливих нечітких розбиттів множини $\Omega$ на $N$ нечітких підмножин через $\mathfrak{R}_{\Omega}^{N}$, тобто:

$$
\mathfrak{R}_{\Omega}^{N}=\left\{\left(\Omega_{1}, \ldots, \Omega_{N}\right): \sum_{i=1}^{N} \mu_{k}(x)=\mu_{\Omega}(x)=1 \forall x \in \Omega\right\} .
$$

Зауважимо, що клас чітких можливих розбиттів $є$ підкласом класу $\mathfrak{R}_{\Omega}^{N}$. У свою чергу, оскільки елемент множини $\mathfrak{R}_{\Omega}^{N} \in$ сукупністю підмножин $\Omega_{1}, \ldots, \Omega_{N}$, кожне з яких належить $\Omega$, то нечітка множина $\mathfrak{R}_{\Omega}^{N} \in$ нечіткою підмножиною множини $\mathrm{X}=\underbrace{\Omega \times \ldots \times \Omega}_{N}$.

Введемо на множині можливих нечітких розбиття $\mathfrak{R}_{\Omega}^{N}$ цільовий функціонал $\left(\mathrm{F}=\mathfrak{R}_{\Omega}^{N} \rightarrow \mathrm{R}^{1}\right)$ у вигляді

$$
\mathrm{F}\left(\left\{\Omega_{1}, \ldots, \Omega_{N}\right\}, \tau\right)=\sum_{i=1}^{N} \int_{\Omega_{i}}\left(c\left(x, \tau_{i}\right) / \omega_{i}+a_{i}\right) \rho(x) d x,
$$

де, як і у чіткій задачі А1, функції $c\left(x, \tau_{i}\right)$ - задані, дійсні, обмежені, визначені на $\Omega \times \Omega$, вимірні за $x$ при будь-якому фіксованому $\tau_{i}=\left(\tau_{i}^{(1)}, \ldots, \tau_{i}^{(n)}\right)$ з $\Omega$ для всіх $i=1, \ldots N ; \rho(x)$ - задана, обмежена, вимірна на $\Omega$, невід'ємна функція; $a_{1}, \ldots, a_{N}$ - задані невід'ємні числа, $\omega_{1}, \ldots, \omega_{N}$ - задані позитивні числа.

На відміну від чіткої задачі А1, тут, по-перше, $\Omega_{i}, i=1, \ldots N$, нечіткі підмножини множини $\Omega$ з заданими координатами типових представників (або 
центрів) $\tau_{i}$, причому кожний центр $\tau_{i}$ належить $\Omega$; по-друге, міра множини межових точок $\Omega_{i}, i=1, \ldots N$, не обов'язково дорівнює нулю.

Тоді нечітким аналогом задачі А1 буде наступна задача.

Задача $A 3$ [16]. Знайти таке розбиття множини $\Omega$ на $N$ нечітких підмножин $\Omega_{1}, \ldots, \Omega_{N}$ (серед яких можуть бути і порожні), яке задовольняє умовам

$$
\bigcup_{i=1}^{N} \Omega_{i}=\Omega, \sup _{x \in \Omega} \mu_{\Omega_{i} \cap \Omega_{j}}(x)<1, i \neq j, i, j=1, \ldots, N,
$$

і в деякому сенсі «мінімізує» цільової функціонал (4). Тут під «мінімізацією» можна розуміти вибір нечіткого розбиття, якому відповідає в деякому сенсі найкраще нечітке значення цільового функціоналу.

Перепишемо задачу А 3 в термінах функції належності $\mu_{i}(\cdot)$ як розширення задачі A1.1 на нечіткий випадок (нечіткий аналог задачі 1.1) наступним чином.

Задача A3.1. (нечіткого оптимального розбиття множини $\Omega$ без обмежень з фіксованими центрами підмножин).

Знайти вектор-функцію $\mu^{*}(\cdot)=\left(\mu_{1}^{*}(\cdot), \ldots, \mu_{N}^{*}(\cdot)\right) \in \Gamma$ таку, що

$$
I\left(\mu^{*}(\cdot)\right)=\min _{\mu(\cdot) \in \Gamma} I(\mu(\cdot)),
$$

де

$$
\begin{gathered}
I(\mu(\cdot))=\int_{\Omega} \sum_{i=1}^{N}\left(\mu_{i}(x)\right)^{m}\left(c\left(x, \tau_{i}\right) / \omega_{i}+a_{i}\right) \rho(x) \mathrm{d} x, \\
\Gamma=\left\{\mu(\cdot)=\left(\mu_{1}(\cdot), \ldots, \mu_{N}(\cdot)\right): \sum_{i=1}^{N} \mu_{i}(x)=1, x \in \Omega, 0 \leq \mu_{\Omega_{i}}(x) \leq 1, i=1, \ldots, N, \quad x \in \Omega\right\},
\end{gathered}
$$

$x=\left(x^{(1)}, \ldots, x^{(n)}\right) \in \Omega ; \quad \tau=\left(\tau_{1}, \ldots, \tau_{N}\right)$ - заданий вектор $3 \Omega^{N} ; \tau_{i}=\left(\tau_{i}^{(1)}, \ldots, \tau_{i}^{(n)}\right)$; $a_{1}, \ldots, a_{N}$ - задані невід'ємні числа; $\omega_{1}, \ldots, \omega_{N}$ - задані позитивні числа; $m$ - параметр експоненціальної ваги [16].

Тут під позначенням «min» розуміється, що $\mu^{*}(\cdot)$ в певному сенсі «мінімізує» цільової функціонал $I(\mu(\cdot))$. Вимога умови (6) виконання рівності обумовлена тією обставиною, що шукане нечітке розбиття $\left(\Omega_{1}, \ldots, \Omega_{N}\right)$ має «покривати» звичайне чітке розбиття $\left(\Omega_{1}, \ldots, \Omega_{N}\right)$, яке $\epsilon$ в той же час елементом нечіткої множини допустимих розв'язків, для якого значення функцій належності кожного з елементів дорівнює 1.

Зауважимо, що якщо необхідно виключити появу порожніх підмножин в шуканому нечіткому розбитті, то до обмежень задачі А3.1 слід додати умову

$$
\int_{\Omega} \mu_{\Omega_{i}}(x) d x>0, \quad i=1, \ldots, N .
$$

Аналогічно викладеному вище можуть бути розширені постановки задач А1.2 і А1.3. Сформулюємо їх. 
Задача A3.2. (нечіткого оптимального розбиття множини $\Omega$ без обмежень з відиуканням координат центрів нечітких підмножин). Знайти

$$
\min _{(\mu(\cdot), \tau) \in \Gamma \times \Omega^{N}} \int_{\Omega}^{N} \sum_{i=1}^{N}\left(c\left(x, \tau_{i}\right) / \omega_{i}+a_{i}\right) \rho(x)\left(\mu_{i}(x)\right)^{m} d x
$$

де

$$
\begin{gathered}
\Gamma=\left\{\mu(x)=\left(\mu_{1}(x), \ldots, \mu_{N}(x)\right): \sum_{i=1}^{N} \mu_{i}(x)=1, x \in \Omega,\right. \\
\left.0 \leq \mu_{i}(x) \leq 1, i=1, \ldots, N, x \in \Omega\right\}, \\
\tau=\left(\tau_{1}, \ldots, \tau_{N}\right) \in \Omega^{N} .
\end{gathered}
$$

Задача A3.3. (нечіткого оптимального розбиття множини $\Omega$ при обмеженнях у формі рівностей та нерівностей з заданими координатами центрів нечітких підмножин). Знайти

$$
\min _{\mu(\cdot) \in \Gamma_{1}} \int_{\Omega} \sum_{i=1}^{N}\left(c\left(x, \tau_{i}\right) / \omega_{i}+a_{i}\right) \rho(x)\left(\mu_{\Omega_{i}}(x)\right)^{m} d x
$$

де

$$
\begin{gathered}
\Gamma_{1}=\left\{\mu(x)=\left(\mu_{\Omega_{1}}(x), \ldots, \mu_{\Omega_{N}}(x)\right) \in \Gamma, x \in \Omega ; \int_{\Omega} \rho(x) \mu_{\Omega_{i}}(x) d x \leq b_{i}, \quad i=1, \ldots, N\right\} ; \\
\Gamma=\left\{\mu(x)=\left(\mu_{\Omega_{1}}(x), \ldots, \mu_{\Omega_{N}}(x)\right): \sum_{i=1}^{N} \mu_{\Omega_{i}}(x)=1, \quad x \in \Omega,\right. \\
\left.0 \leq \mu_{\Omega_{i}}(x) \leq 1, i=1, \ldots, N, x \in \Omega\right\}, \tau=\left(\tau_{1}, \ldots, \tau_{N}\right) \in \Omega^{N} .
\end{gathered}
$$

\section{4 Основні напрями теоретичних застосувань математичної теорії оп- тимального розбиття множин}

Теорія оптимального розбиття множин ефективно застосовується для розв'язання широкого спектру різних за своєю природою теоретичних і практичних класів оптимізаційних задач, які зводяться в математичній постановці до неперервних моделей оптимального розбиття множин. Наведемо деякі 3 цих класів задач теорії оптимізації.

1. Задачі розпізнавання образів (чітких і нечітких) з метою мінімізації середньої функції втрат від неправильного розпізнавання [13].

2. Задачі теорії класифікації та кластеризації, що вивчає питання розбиття заданої множини (скінченновимірної або нескінченновимірної) елементів на неперетинні підмножини.

3. Задачі, які виникають в теорії статистичних рішень при розбитті простору ознак на класи, що не перетинаються (узагальнена задача НейманаПірсона) [29].

4. Задачі глобальної оптимізації [30, 31].

5. Задачі побудови оптимальних квадратур [32].

6. Неперервні задачі оптимального кульового (однократного та багатократного) покриття обмежених множин евклідового простору [19].

7. Задачі геометричного проектування.

8. Задачі цілочисельного стохастичного програмування. 
9. Задачі стартового керування параболічною системою [18].

10. Задачі ідентифікації багатозонних моделей динаміки, що відносяться до класу обернених задач, сформульованих для систем із зосередженими пара- метрами [20].

11. Задачі побудови діаграми Вороного та її узагальнень [33, 34].

Продемонструємо можливість застосування математичного апарату розв'язання неперервних задач ОРМ до задач побудови діаграми Вороного та iї узагальнень.

\section{5 Теорія оптимального розбиття як універсальний математичний апа- рат побудови діаграми Вороного і її узагальнень}

Математична теорія оптимального розбиття множин на сьогодні $є$ потужним інструментом для розв'язання багатьох теоретичних і практично важливих задач, що зводяться в математичній постановці до неперервних задач оптимального розбиття множини з евклідового простору (лінійних або нелінійних, статичних або динамічних, детермінованих або стохастичних, в умовах визначеності або нечітких ). Розв'язок ряду модельних задач зі згаданих класів часто призводить до математичних об'єктів, які мають назву діаграми Вороного або розбиття Діріхле [1]. Нестрого діаграма Вороного описується наступним чином.

Якщо на площині заданий скінчений набір точок (точок-генераторів), то для кожного елемента набору можна визначити так звану комірку Вороного множину точок площині, для яких даний елемент $є$ найближчим з усіх точок набору. Межі комірок Вороного утворюють розбиття площини, яке і називають діаграмою Вороного. Наведена математична структура називається по імені Георгія Вороного [35], але вона також відома під назвою полігонів Тіссена [36] або двовимірних комірок Діріхле [37].

Діаграми Вороного в двох і трьох вимірах використовуються в самих різних областях прикладних наук: кристалографії, фізики, астрономії, хімії, мікробіології, комп'ютерній графіці, при вирішенні проблем штучного інтелекту, розпізнавання образів, офтальмології. У разі загальних метричних просторів комірки часто називають метричними фундаментальними багатокутниками. Інші еквівалентні назви цієї математичної структури (або конкретних важливих iï додатків) - багатогранники Вороного, полігони Вороного, домени впливу, розкладання Вороного, мозаїки Вороного, тесселяції Діріхле. Слід зауважити, що під комірками розуміються виділені частини континуальної системи, які періодично розташовані по об'єму системи, при цьому кожна частка або вакансія займає модифіковану комірку Вигнера-Зейтца, оточену поліедром Вороного [38]. $€$ й інші області застосування розбиття ДіріхлеВороного. Змістовні огляди застосувань діаграм Вороного в різних областях приведені в роботі [39].

Діаграми Вороного - досить добре вивчений об'єкт, і для них отримано багато різних алгоритмів, що працюють за оптимальну асимптотику $O(N \log (N))$, а деякі $з$ цих алгоритмів навіть працюють в середньому за 
$O(N)$. Однак всі ці алгоритми досить складні. А що стосується діаграми Вороного на випадок простору довільної розмірності, то її побудова пов'язана зі значними алгоритмічними проблемами. Дійсно, відомо [39], що при заданій кількості точок $N$ кількість елементів, необхідних для опису діаграми Вороного, зростає експоненціально в залежності від розмірності простору.

Математичним апаратом побудови діаграм Вороного, який має ряд переваг у порівнянні з відомими підходами, описаними в науковій літературі, є теорія оптимального розбиття множин, яка розроблена О.М. Кісельовою [1]. У роботах $[33,34]$ на основі методів теорії ОРМ запропоновані алгоритми побудови як стандартної діаграми Вороного з чіткими параметрами, так і різних їі узагальнень. В $[33,34]$ показано, що при відповідному формулюванні неперервної лінійної задачі оптимального розбиття множини розв'язок цієї задачі призводить до того чи іншого варіанту діаграми Вороного заданої кількості точок. При цьому алгоритми розв'язання неперервних лінійних задач ОРМ не залежить від розмірності евклідового простору $E_{n}$, що містить обмежену множину, що підлягає розбиттю на підмножини; не залежить від геометрії множини, що підлягає розбиттю; складність реалізації алгоритмів побудови діаграм Вороного на основі методів теорії ОРМ не збільшується при збільшенні кількості $N$ точок-генераторів, а швидкодія допоміжних ітераційних процедур недиференційовної оптимізації дозволяє розв'язувати задачі великих розмірностей $(N=100,200,300$ і т.д.); алгоритми можуть бути застосовні до побудови не тільки діаграм Вороного заданої кількості точок-генераторів 3 фіксованим їх розташуванням, а й з оптимальним розміщенням цих точок в обмеженій множині простору $E_{n}$. Запропонований в роботах $[40,41]$ підхід має високий ступінь універсальності, оскільки дає можливість легко будувати не тільки вже відомі діаграми Вороного, а й конструювати нові. Зокрема, моделі і методи розв'язання неперервних задач ОРМ можуть бути узагальнені на випадки нечіткого завдання вихідних параметрів задач або вимоги нечіткого розбиття множини, в результаті чого результуючі діаграми Вороного також можуть мати нечіткий характер (нечіткі діаграми Вороного).

За аналогією з класифікацією нечітких задач ОРМ можна виділити два основних типи нечітких діаграм Вороного: діаграми Вороного 3 нечіткими параметрами і діаграми Вороного, в яких множина точок, що утворюють комірки Вороного, є нечіткими множинами (нечіткі комірки). А розв'язок нечітких задач ОРМ, як і для детермінованих задач ОРМ, призводить до побудови нечітких діаграм Вороного двох основних типів: діаграми Вороного з нечіткими параметрами і діаграми з нечіткими комірками Вороного.

В роботі [42] описаний алгоритм побудови мультиплікативно зваженої діаграми Вороного при наявності нечітких параметрів 3 оптимальним розміщенням скінченої кількості N точок-генераторів в обмеженій множині $\Omega 3 n$ вимірного евклідового простору $E_{n}(n \geq 2)$. Алгоритм розроблений на основі синтезу методів розв'язання задач теорії ОРМ [10] з нейронечіткого технологіями [12] і модифікаціями -алгоритми Н.З. Шора для розв'язання негладких 
задач оптимізації [26]. У роботі [16] запропонований алгоритм побудови одного $з$ варіантів нечітких діаграм Вороного, коли множина точок, що утворюють комірку Вороного, може бути нечіткою. Алгоритм розроблений на основі синтезу методів теорії оптимального розбиття множин та теорії нечітких множин. Універсальність пропонованого підходу до побудови діаграм Вороного підтверджується ще і тим, що моделі і методи рішення неперервних задач ОРМ можуть бути узагальнені на випадок нечіткого завдання вихідних параметрів задач або вимоги нечіткого розбиття множини, в результаті чого і результуючі діаграми Вороного можуть носити нечіткий характер.

\section{3. Аналіз отриманих результатів}

3 викладеного вище витікає, що при формулюванні відповідної неперервної задачі ОРМ розв'язок цієї задачі призводить до того чи іншого варіанту діаграми Вороного заданого числа точок. У табл. 1 наведено відповідність між конкретним варіантом діаграми Вороного і математичною моделлю неперервної задачі ОРМ, в результаті розв'язання якої може бути отримана ця діаграма.

Таблиияя 1

Моделі неперервних задач ОРМ і діаграми Вороного, які с оптимальним розв'язком цих задач

\begin{tabular}{|c|c|c|}
\hline \multicolumn{2}{|l|}{ Модель неперервної задачі ОРМ } & Діаграма Вороного \\
\hline \multicolumn{3}{|c|}{ Задача A1.1. : $\rho(x)=1 x \forall \Omega$} \\
\hline \multicolumn{2}{|l|}{$c\left(x, \tau_{i}\right)=r\left(x, \tau_{i}\right), a_{i}=0 \quad i=1, \ldots, N$} & Стандартна \\
\hline \multicolumn{2}{|l|}{$c\left(x, \tau_{i}\right)=r\left(x, \tau_{i}\right), a_{i} \neq 0, \quad i=1, \ldots, N$} & Адитивно зважена \\
\hline \multicolumn{2}{|l|}{$c\left(x, \tau_{i}\right)=r\left(x, \tau_{i}\right) / w_{i}, w_{i}>0, a_{i}=0, i=1, \ldots, N$} & Мультиплікативно зважена \\
\hline \multicolumn{2}{|l|}{$c\left(x, \tau_{i}\right)=\beta_{i} r\left(x, \tau_{i}\right), \beta_{i}, \mathrm{a}_{i}>0, \quad i=1, \ldots, N$} & Мультиплікативно і адитивно зважена \\
\hline \multicolumn{2}{|l|}{$c\left(x, \tau_{i}\right)=r^{2}\left(x, \tau_{i}\right), a_{i}=A_{i}^{2}, i=1, \ldots, N$} & $\begin{array}{l}\text { Діаграма Пауэра або Лагерра (Power } \\
\text { Voronoi Diagram) } 3 \text { вагами } A_{i}^{2}\end{array}$ \\
\hline \multicolumn{2}{|l|}{$c\left(x, \tau_{i}\right)=-r\left(x, \tau_{i}\right), a_{i}=0, \quad i=1, \ldots, N$} & Дальньої точки \\
\hline \multicolumn{2}{|l|}{$c\left(x, \tau_{i}\right)=f_{i}\left(x, \tau_{i}\right), \quad a_{i}=0, \quad i=1, \ldots, N$} & Заснована на ефективності \\
\hline \multicolumn{2}{|l|}{$\begin{array}{l}\text { Задача A1.1: } \rho(x)-\text { довільна } \\
c\left(x, \tau_{i}\right)=r\left(x, \tau_{i}\right), a_{i}=\text { const }, \quad i=1, \ldots, N\end{array}$} & Інші зважені діаграми \\
\hline \multicolumn{3}{|c|}{ Задача A1.2. : $\rho(x)=1 x \forall \Omega$} \\
\hline$c\left(x, \tau_{i}\right)=r\left(x, \tau_{i}\right), a_{i}=0, i=1, \ldots, N$ & \multicolumn{2}{|c|}{$\begin{array}{c}\text { Скінченої кількості точок-генераторів, оптимально } \\
\text { розміщених в } \Omega\end{array}$} \\
\hline$c\left(x, \tau_{i}\right)=r\left(x, \tau_{i}\right), \quad a_{i} \neq 0, \quad i=1, \ldots, N$ & \multicolumn{2}{|c|}{$\begin{array}{l}\text { Адитивно зважена скінченої кількості точок- } \\
\text { генераторів, оптимально розміщених } \Omega\end{array}$} \\
\hline \multicolumn{3}{|c|}{ Задача A1.3. : $\rho(x)=1 x \forall \Omega$} \\
\hline \multicolumn{2}{|c|}{$c\left(x, \tau_{i}\right)=r\left(x, \tau_{i}\right), \quad a_{i}=0, \quad b_{i} \neq 0, i=1, \ldots, N$} & $\begin{array}{c}3 \text { обмеженнями на потужності } \\
\text { точок-генераторів }\end{array}$ \\
\hline \multicolumn{2}{|l|}{$c\left(x, \tau_{i}\right)=r\left(x, \tau_{i}\right), \quad a_{i} \neq 0, \quad b_{i} \neq 0, i=1, \ldots, N$} & $\begin{array}{c}\text { Адитивно зважена з обмеженнями на по- } \\
\text { тужності точок-генераторів }\end{array}$ \\
\hline \multicolumn{2}{|c|}{$c\left(x, \tau_{i}\right)=r\left(x, \tau_{i}\right) / w_{i}, w_{i}>0, a_{i} \neq 0, b_{i} \neq 0, \quad i=1, \ldots, N$} & $\begin{array}{c}\text { Мультиплікативно та адитивно зважена } 3 \\
\text { обмеженнями на потужності точок- } \\
\text { генераторів }\end{array}$ \\
\hline
\end{tabular}


ISSN 2074-5893 Питання прикладної математики і математичного моделювання. Випуск 21

Продовження таблиці

\begin{tabular}{|c|c|}
\hline $\begin{array}{l}\text { 3адача A2.1. } \rho(x)=1 x \forall \Omega, \\
\qquad\left(x, \tau_{i}\right)=r\left(x, \tau_{i}\right) / \tilde{w}_{i}, \tilde{w}_{i}>0, \tilde{a}_{i} \neq 0, i=1, \ldots, N\end{array}$ & $\begin{array}{c}\text { Мультиплікативно та адитивно зважена } 3 \\
\text { нечіткими параметрами }\end{array}$ \\
\hline $\begin{array}{l}\text { 3aдача A3.1. } \rho(x)=1 x \forall \Omega, c\left(x, \tau_{i}\right)=r\left(x, \tau_{i}\right), a_{i}=0 \\
i=1, \ldots, N\end{array}$ & $\begin{array}{c}3 \text { нечіткими комірками при заданих коор- } \\
\text { динатах скінченої кількості точок- } \\
\text { генераторів }\end{array}$ \\
\hline $\begin{array}{l}\text { 3адача A3.2. } \rho(x)=1 x \forall \Omega, \\
c\left(x, \tau_{i}\right)=r\left(x, \tau_{i}\right), \quad a_{i}=0, \quad b_{i} \neq 0, i=1, \ldots, N\end{array}$ & $\begin{array}{c}3 \text { нечіткими комірками при заданих коор- } \\
\text { динатах точок-генераторів і обмеженнях на } \\
\text { ix потужності }\end{array}$ \\
\hline $\begin{array}{l}\text { 3adaча A3.3. } \rho(x)=1 x \forall \Omega, c\left(x, \tau_{i}\right)=r\left(x, \tau_{i}\right), a_{i}=0 \text {, } \\
i=1, \ldots, N\end{array}$ & $\begin{array}{c}3 \text { нечіткими комірками скінченої кількості } \\
\text { точок-генераторів, оптимально розміщених } \\
\text { в } \Omega\end{array}$ \\
\hline
\end{tabular}

Табл. 1 можна продовжити, змінюючи параметри задач ОРМ і отримуючи при цьому все нові, не представлені в науковій літературі, узагальнення діаграми Вороного.

Висновки. Теорія оптимального розбиття множин $є$ напрямком сучасної теорії оптимізації, який активно розвивається і ефективно застосовується для розв'язання широкого спектра різних за своєю природою теоретичних і практичних класів оптимізаційних задач, що зводяться в математичній постановці до неперервних моделей оптимального розбиття множин. Це задачі розпізнавання образів (чітких і нечітких) з метою мінімізації середньої функції втрат від неправильного розпізнавання; задачі теорії класифікації та кластеризації, яка досліджує питання розбиття заданої множини (скінченновимірної або нескінченновимірної) елементів на підмножини, що не перетинаються; задачі, що виникають в теорії статистичних рішень при розбитті простору ознак на класи, що не перетинаються, (узагальнена задача НейманаПірсона) та багато інших.

Показана можливість застосування математичної теорії оптимального розбиття множин до побудови діаграми Вороного і різних ії узагальнень. Математичний та алгоритмічний апарат побудови різних варіантів діаграми Вороного заснований на формулюванні неперервних задач оптимального розбиття множин 3 критеріями якості розбиття, що забезпечують відповідні види діаграми Вороного.

Універсальність даного підходу до побудови діаграм Вороного підтверджується ще і наступними фактами:

- моделі і методи розв'язання неперервних задач оптимального розбиття множин можуть бути узагальнені на випадок нечіткого завдання вихідних параметрів задачі або вимоги нечіткого розбиття множини, в результаті чого і результуючі діаграми Вороного можуть носити нечіткий характер;

- серед неперервних задач оптимального розбиття множин виділяють клас задач, в яких поряд з розбиттям потрібно відшукати ще й оптимальне розташування центрів підмножин. Таким чином, одночасно із задачею побудови діаграми Вороного можна ставити задачу відшукання оптимальних (в якомусь сенсі) координат точок-генераторів цієї діаграми;

- складність реалізації алгоритмів побудови діаграм Вороного на основі методів математичної теорії оптимального розбиття істотно не змінюється при збільшенні кількості точок-генераторів; 
- результатом такого підходу є можливість будувати не тільки вже відомі діаграми Вороного, а й конструювати нові.

\section{Бібліографічні посилання}

1. Kiseleva E.M.: The Emergence and Formation of the Theory of Optimal Set Partitioning for Sets of the n-Dimensional Euclidean Space. Theory and Application. Journal of Automation and Information Sciences. 2018. 50(9). P. 1-24. https://doi.org/JAutomatInfScien. v50.i9.10.

2. Киселева Е.М. Математические методы оптимального разбиения множеств и их приложения. Дн-ск: ДГУ, 1982. 108 с.

3. Кісельова О.М. Становлення та розвиток теорії оптимального розбиття множин. Теоретичні і практичні застосування: монографія. Дніпро: Ліра. ДНУ, 2018. 532 с.

4. Kiseleva E.M., Shor N.Z. Analysis of algorithms for a class of continuous partition problems. Cybernetics and Systems Analysis. 1994. 30 (1). P. 64-74.

5. Kiseleva E.M., Zhiltsova A.A. The Necessary Optimality Conditions for Continuous Problems of Set Partitioning in Terms of the Theory of Set Functions. Journal of Automation and Information Sciences. 2008.40 (12). P.14-26

6. Kiseleva E.M., Kadochnikova Ya.E. Solving a continuous single-product problem of optimal partitioning with additional conditions. Journal of Automation and Information Sciences, 2009. 41 (7). P. 48-63.

7. Kiselyova O.M., Dunaichuk M.S. Soling a Continuous Nonlinear Problem of Optimal set Partition with Arrangement of Subset Centers in the Case of a Convex Objective Functional. Cybernetics and Systems Analysis. 2008. 44 (2). P. 261-275.

8. Kiseleva E.M., Zhiltsova A.A., Stroyeva V.A. General scheme to obtain necessary optimality conditions for continuous optimal set partitioning problems. Journal of Automation and Information Sciences. 2012. 44 (9). P. 51-65.

9. Киселева Е.М., Строева В.А. Алгоритм решения нелинейной непрерывной многопродуктовой задачи оптимального разбиения множеств с размещением центров подмножеств Проблемы управления и информатики. 2012. №1. С. 40-53.

10. Киселева Е.М., Шор Н.З. Непрерывные задачи оптимального разбиения множеств: теория, алгоритмы, приложения. К.: Наукова думка, 2005. 564 с.

11. Kiseleva E., Prytomanova O., Zhuravel S. An algorithm for solving location-allocation problem with fuzzy parameters. Scientific discussion. Praha (Czech Republic). 2020. 1 (40). P. 11-18.

12. Kiseleva E.M., Prytomanova O.M., Zhuravel S.V.: Algorithm for Solving a Continuous Problem of Optimal Partitioning with Neurolinguistic Identification of Functions in Target Functional. Journal of Automation and Information Science. 2018. 50(3). P. 1-20. https://doi.org/10.1615/JAutomatInfScien.v50.i3.10.

13. Киселева Е.М., Коряшкина Л.С., Ус С.А. Теория оптимального разбиения множеств в задачах распознавания образов, анализа и идентификации систем. Дніпро: НГУ, 2015. $270 \mathrm{c}$.

14. Кісельова О.М., Притоманова О.М., Журавель С.В., Шаравара В.В. Алгоритм розв'язання однієї задачі оптимального розбиття з нечіткими параметрами в цільовому функціоналі. Питання прикладної математики і математичного моделювання. 2018. Вип. 18. С. 85-98.

15. Притоманова О.М. Розв'язання задачі оптимального розбиття множин із нечіткими параметрами в обмеженнях. Вісник Львівського національного університету. Серія прикладна математика та інформатика,2019. Вип. 27. С. 97-107.

16. Кісельова, О.М., Гарт, Л.Л., Притоманова, О.М., Балейко, Н.В. Нечіткі задачі оптимального розбиття множин: теоретичні основи, алгоритми, застосування: монографія. Дніпро: Лipa, 2020. 400 с. 
17. Kiseleva E.M., Koriashkina L.S., Shevchenko T.A. Solving the Dynamic Optimal Set Partitioning Problem with Arrangement of Centers of Subsets. Cybernetics and Systems Analysis. 2014. 50 (6). P. 842-853.

18. Киселева Е.М., Коряшкина Л.С. Модели и методы решения непрерывных задач оптимального разбиения множеств: линейные, нелинейные, динамические Киев: Наукова думка, 2013. 606 c.

19. Kiseleva E.M., Lozovskaya L.I., Timoshenko E.V. Solution of continuous problems of optimal covering with spheres using optimal set-partition theory. Cybernetics and Systems Analysis. 2009. 45 (3). P. 421-437.

20. Киселева Е.М., Коряшкина Л.С. Непрерывные задачи оптимального разбиения множеств и $r$-алгоритмы. Киев: Наукова думка, 2015. 400 с.

21. Kiseleva E., Prytomanova O. An algorithm for solving fuzzy optimal partitioning of set problem with location of centers of the subsets. Znanstvena misel journal. Ljubljana (Slovenia). 2020. 1 (40). P. 19-26.

22. Kiseleva E., Prytomanova O., Hart L. Solving a Two-stage Continuous-discrete Problem of Optimal Partitioning-Allocation with the Subsets Centers Placement. Open Computer Science. De Gruyter. 2020. Vol 10. P. 124-136.

23. Киселева Е.М., Притоманова О.М., Ус С.А. Решение двухэтапной непрерывнодискретной задачи оптимального разбиения-распределения с заданным положением центров подмножеств. Кибернетика и системный анализ. 2020. №1. С. 3-15.

24. Кісельова О.М, Притоманова О.М., Дзюба С.В., Падалко В.Г. Розв'язання двоетапної неперервно-дискретної задачі оптимального розбиття-розподілу з нечіткими параметрами. Питання прикладної математики і математичного моделювання. 2019. Вип. 19. C. $106-116$.

25. Кісельова О.М., Притоманова О.М. Нечітка задача оптимального розбиття множин із обмеженнями на розміщення центрів підмножин. Системні дослідження та інформаиійні технологїі. 2020. №1. С. 78-89.

26. Shor N.Z. Nondifferentiable optimization and polynomial problems. Boston; Dordrecht; London: Kluwer Acad. Publ., 1998. 412 p.

27. Stetsyuk P.I. Shor's r-Algorithms: Theory and Practice. In: Optimization Methods and Applications: In Honor of the 80th Birthday of Ivan V. Sergienko. Ed. by Butenko S., Pardalos P.M, Shylo V. Springer, 2017. P. 495-520.

28. Zadeh L.A.: Fuzzy sets. Information and Control. 1965. No 8. P. 338-353.

29. Киселева Е.М. Решение обобщенной задачи Неймана-Пирсона с использованием методов оптимального разбиения множеств. Журнал вычислительной математики и математической физики. 1992. 31 (1). С. 167-173.

30. Kiseleva E.M., Stepanchuk T. On the Efficiency of a Global Non-differentiable Optimization Algorithm Based on the Method of Optimal Set Partitioning. Journal of Global Optimization. 2003. V. 25. P. 209-235.

31. Киселева Е.М., Степанчук Т.Ф. Поиск глобального минимума недифференцируемой функции с помощью метода оптимального разбиения множеств. Проблемы управления и информатики. 2002. №2. С. 45-60.

32. Киселева Е.М., Степанчук Т.Ф. О выборе оптимальных коэффициентов и оптимальных узлов квадратурных формул для функциональных классов, заданных квазиметриками. Проблемы управления и информатики. 2002. №3. С. 138-153.

33. Kiseleva E.M., Koriashkina L.S. Theory of continuous optimal set partitioning problems as a universal mathematical formalism for constructing Voronoi diagrams and their generalizations I. Theoretical foundations, Cybernetics and Systems Analysis. 2015. 51(3). P. 325-335. https://doi.org/10.1007/s10559-015-9725-x.

34. Kiseleva E.M., Koriashkina L.S. Theory of continuous optimal set partitioning problems as a universal mathematical formalism for constructing voronoi diagrams and their 
generalizations. II. Algorithms for constructing Voronoi diagrams based on the theory of optimal set partitioning. Cybernetics and Systems Analysis. 2015. 51(4). P. 489-499. https://doi.org/10.1007/s10559-015-9740-y.

35. Voronoi Georgy. Nouvelles applications des parametres continus ala theorie des formes quadratiques. Journal fur die Reine und Angewandte Mathematik. 1907. P. 97-178.

36. Thiessen A.H. Precipitation averages for large areas. Monthly Weather Review. 1911. 39(7). P. 1082-1084.

37. Fejes, T. G. Multiple packing and covering of spheres. Acta Math. Acad. Sci. Hungar. 1979. 34 (1-2). P. 165-176.

38. Yoshiaki O. A. geometrical solution for quadratic bicriteria location models. European Journal of Operational Research. 1999. No 114. P. 380-388.

39. Preparata F., Sheimos, M. Computational geometry: an introduction. Springer. First Edition edition, 1993. $390 \mathrm{p}$.

40. Киселева Е.М., Гарт Л.Л., Притоманова О.М. Алгоритм построения диаграмм Вороного с оптимальным размещением точек-генераторов на основе теории оптимального разбиения множеств. Проблемы управления и інформатики. 2020. №2 . С. 5-15.

41. Kiseleva E.M., Hart L.L., Prytomanova O.M., Zhuravel S.V. Construction of a generalized Voronoi diagram with optimal placement of generator points based on the theory of optimal set partitioning. Matematychni Studii. 2020.53 (1). P. 109-112.

42. Kiseleva E., Prytomanova O., Padalko V. Chapter "An Algorithm for constructing additive and multiplicative Voronoi Diagrams Under Uncertainty" in Lecture Notes in Computational Intelligence and Decision Making. Springer, 2021. Vol. 1246. P. 714-727.

Надійшла до редколегії 26.08.2021. 